\title{
Urgences
}

\section{Du romantisme au crayon feutre}

\section{Louise Blouin}

Numéro 26, décembre 1989

Des textes qui chantent

URI : https://id.erudit.org/iderudit/025557ar

DOI : https://doi.org/10.7202/025557ar

Aller au sommaire du numéro

Éditeur(s)

Urgences

ISSN

0226-9554 (imprimé)

1927-3924 (numérique)

Découvrir la revue

Citer cet article

Blouin, L. (1989). Du romantisme au crayon feutre. Urgences, (26), 74-81.

https://doi.org/10.7202/025557ar d'utilisation que vous pouvez consulter en ligne.

https://apropos.erudit.org/fr/usagers/politique-dutilisation/ 


\title{
Du romantisme au crayon feutre Louise Blouin
}

\author{
Café RImbaud 1
}

Je t'attends au café Rimbaud Je sais que tu ne viendras pas J'écris dans un carnet de notes Une mélodie au crayon feutre

5 Une chanson pour la radio

Demain, je serai toujours là A la même table qu'autrefois J'aurai écrit une symphonie Un concert pour boîte à musique

10 Je t'attends au Café Rimbaud Je sais que tu ne viendras pas J'écris dans un carnet de notes Une mélodie au crayon feutre Une chanson pour la radio

15 Demain je devrai m'en aller Avec mes souvenirs rétros Et mes visions au fond des yeux Comme des images d'Épinal J'ai laissé au Cafó Rimbaud

20 Une page de mon carnet de notes Je sais tu ne la liras pas Je t'écris un space-opéra Dans un hôtel tout près du ciel Au bout du monde en t'attendant

On sait que la chanson est classée par les spécialistes dans la catégorie "paralittérature». Cependant, tous les spécialistes de cette littérature parallèle ont aussi analysé certains romans dits très * littéraires" et y ont identifié des stratégies pour fasciner et pour attirer les lecteurs et ainsi

1 Album ou cassette, Radio-Canada, SP 008, 1987. Paroles: Lucien Francosur; musique: cinq versions... Marie Bernard, Gerry Boulet, François Cousineau, Steve Faulkner, Michel Rivard. Paroles dejà publiées dans Rock-désir, Montréal, VLB éditeur, 1984. 
devenir "populaires ». Au nom de la rose de Umberto Eco, par exemple, possède une trame policière solide, capable d'appâter le public. En plus de son suspense, ce roman propose aussi de longs passages en latin qui, de prime abord, ne font pas tellement grand public, ne happent pas le lecteur moyen. Ainsi, la frontière peut parfois s'avérer bien mince entre le «littéraire» et le " paralittéraire».

Cafe Rimbaud de Lucien Francour se veut une chanson et elle ne se prend pas pour un poème; pourtant, quelques indices nous plongent dans le littéraire à l'occasion.

Le titre évoque un grand poète devenu mythique, Arthur Rimbaud, pour lequel Francœur entretient une certaine passion, frôlant parfois l'envie (Si Rimbaud pouvait me lire, Saint-Lambert, Noroît). N'oublions pas, même si c'est une lapalissade de le souligner, que Rimbaud est français. Nous sommes en Amérique du Nord, continent que Francœur revendique comme lieu d'appartenance, en langue française, même s'il ne craint jamais d'assaisonner avec un peu d'américain, pour souligner notre proximité avec les États-Unis et le fait que nous baignons dans les manifestations langagières anglophones. Mais cette chanson laisse deviner plutôt une extrême fascination exercée par la France et par l'écriture.

Juxtaposé à «Rimbaud», il y a le mot «Café»; ceci suggère un endroit calme, français encore, ou québécois francophone, où se côtoient bohèmes, intellectuels, artistes, écrivains. En France, on lit et on écrit dans les cafés, car les appartements sont souvent petits, et puis au "Café de Flore", "Aux deux magots » ou ailleurs, on a l'impression de percevoir les effluves des grands auteurs du passé. Bernanos, par exemple, a écrit ses romans dans des cafés, et cela se perpétue encore aujourd'hui.

Aucune incidence américaine n'est donc présente dans le titre, mais on y trouve une référence à la culture européenne, en l'occurrence française. De plus, cette allusion nous relie au dix-neuvième siècle, époque du romantisme et des bouleversements de l'écriture. C'est un siècle évidemment très dense en roman comme en poésie. La thématique de la chanson rejoint certaines idéologies du siècle du romantisme, tant par son idéalisme que par son lyrisme.

D'abord, on y parle au «je», puis on est patient, tenace, insistant, dans la quête amoureuse, fût-elle impossible, voire vouée irrémédiablement à l'échec («Demain je serai toujours 
76

là", v. 6). On ressent la tristesse et le désespoir, liés à la lucidité, accusée par le vers \&Je sais que tu ne viendras pas * (v. 11). Cette chanson ressemble à une lettre et la correspondance entre amants, qui expriment leurs désirs transis par la cruauté de l'absence, occupe une place privilégiée dans la littérature du dix-neuvième siècle.

Le mode d'écriture se révèle proche du geste physique, d'un certain effort que cela prend pour aligner des mots, surtout des émotions. Ainsi, Francœur ne parle pas d'ordinateur, mais d'un carnet de notes - ici encore l'image du poète qui traîne ses cahiers, écrit sur des napperons, comme une sorte de nomade. Il utilise un crayon plutôt qu'une plume ou une machine à écrire; voilà un outil simple et rudimentaire, relié a l'enfance, au geste concret de faire des lettres (et de faire une lettre). Il y a donc une acceptation du rythme lent de l'écriture. Prendre le temps de dire avant de chanter.

"Feutre" signifie une certaine douceur, une certaine intimité et aussi les techniques modernes de fabrication d'instruments d'écriture. Aussi, il n'écrit pas une prose, mais une chanson. Et là réside le souci de rejoindre l'autre efficacement et facilement: une chanson que tous pourront entendre et apprécier, car elle exprime des sentiments éprouvés universellement, le désir et l'attente de l'être aimé. Donc, ces mots pourront tourner à la radio et, par leur accessibilité, ils nous ramènent à la paralittérature.

Cette chanson se voit liée au romantisme par son côté grandiose, puisqu'elle sera "symphonie * et " concert *. Mais il ne faut perdre ni le lecteur ni le ton intimiste, et l'habileté du poète-chanteur réside dans le fait d'associer * concert * et * boîte à musique» (v. 9), comme si la seconde venait, par la miniaturisation qu'elle propose, compenser l'exagération des expressions précédentes et ramener l'émotion au réel. De plus, cette "boîte à musique " suggère encore le dix-neuvième siècle, mais aussi des airs simples, aisés à retenir, touchants. Elle correspond évidemment, dans une autre dimension, aux appareilsradios, créés au vingtième siècle.

Cette métaphore filée (le dix-neuvième siècle) se retrouve aussi dans les échos des mots * souvenirs rétros * et *images d'Épinal». Bien sûr, "souvenirs rétros" propose une redondance, sinon un pléonasme, tandis qu'«images d'Épinal » nous plonge vers la fin du dix-huitième siècle, alors qu'en cette ville 
est constitué un centre d'imagerie populaire (encore un rapport au paralittéraire). Même chez Proust, le narrateur, dans $\dot{A}$ la recherche $d u$ temps perdu, regarde sa lanterne où défilent des images naïves, rattachées au bonheur. * Épinal „, certes, n'est pas aujourd'hui un mot à signification immédiate pour tous, et François Cousineau, par exemple, n'en connaissait pas la signification au moment de mettre en musique ce texte.

Ainsi, cette comparaison correspond aux phrases latines dans $A u$ nom de la rose. Mais l'auteur rattrape son époque, et cette réalité, ancêtre de la bande dessinée (toujours la paralittérature), se métamorphose en "space-opéra * (v. 22). Ce genre moderne fait songer à une sorte de revue rock, proche de la caricature des dessins animés (pensons à Barbarella). «Space *, mot anglais, juxtaposé à "opéra», écrit en français: voilà nos deux réalités quotidiennes à nouveau confrontées. L'auteur ne choisit pas qui l'emportera!

Le mot «space» fait appel à l'univers, à la galaxie, tandis que le mot qui lui est accolé rappelle le dramatique, l'épanchement lyrique (dans les deux sens du terme) des sentiments. Une telle formulation, de même que le reste du texte, combine à nouveau les réalités des deux derniers siècles. Francœur quittera le Café pour * un hôtel près du ciel», façon élégante de parler d'un gratte-ciel. Même rendu au vingtième siècle, par ce changement de lieu et de mots, il demeure dans l'attente, dans le désir: les états d'âme ne changent pas. L'hôtel, comme le café, est un lieu de passage, une escale pour nomades.

Le nomadisme du rockeur est empreint de contradictions. Il va à l'hôtel et au café, ne fait aucune allusion à une maison, à un chez-soi, mais par ailleurs, il revient au même café le lendemain et accepte la situation d'attente dans ce lieu qui devient son territoire, tout en étant un lieu de passage où l'on ne peut demeurer de façon permanente. Seule sa fidélité à son sentiment devient ici sédentaire en se choisissant un site où l'émotion se reconnaît dans son propre miroir, où la nostalgie se réhabite immédiatement avec certitude. Encore de l'idéalisme romantique!

En disant sa vulnérabilité, le « je * du texte se dégage d'une thématique machiste, attribuée au genre classique du rockeur. Sa métamorphose la plus intense, son véritable voyage, s'opère cependant par l'écriture. Avant même d'attendre et d'aimer, il écrit la chanson, possédé par les mots. La création, non 
seulement transforme son quotidien, mais elle en fait partie intégrante et, en quelque sorte, elle en tient lieu, le rêve remplaçant le réel.

Que ce soit chanson, poème, lettre ou space-opéra, tout passe par les phrases et leur musique. Si l'amour s'envole, reste au moins la feuille sur la table du café et l'éternité du texte. D'une émotion, l'écrivain fait une mémoire.

Si le texte peut s'interpréter en fonction de certains aspects relatifs au dix-neuvième et au vingtième siècles, on pourrait aussi s'interroger sur le genre musical possible à greffer sur ce texte. C'est un peu la question qui a été proposée à quelques compositeurs, puisque cette page de Lucien Francœur a été soumise au concours de la chanson francophone, organisé par Radio-Canada, dont elle a remporté le premier prix qui a engendré sa création musicale par François Cousineau, Steve "Cassonade» Faulkner, Gerry Boulet, Michel Rivard et Marie Bernard.

Ceux-ci ont tenté d'inscrire leur vision du texte en notes et en rythme. Chacun a interprété sa version, sauf François Cousineau qui a confié la sienne à l'Acadienne Lina Boudreau. Suite à ce concours, eut lieu une émission animée par l'organisateur Jean-François Doré, heure pendant laquelle celui-ci interroge chaque compositeur (sauf François Cousineau, remplacé par son interprète) sur leur approche, leur compréhension du texte. L'ensemble nous renseigne sur la thématique, les sentiments, les mots privilégiés par chacun.

Évidemment, on entend également chacune des versions et les commentaires des autres compositeurs, de l'animateur et de l'auteur qui découvrent ensemble toutes ces chansons. Ainsi, Café Rimbaud est d'abord un poème, quand Marcel Sabourin le lit à haute voix, se tenant près du moment d'écriture de Lucien Francour, puis il se métamorphose en prétexte, lorsque les musiciens le prennent en main.

Steve Faulkner exprime sa nervosité, parce que, pour la première fois en quinze ans, il écrit sur le texte de quelqu'un d'autre. Comme les autres, il avait quinze jours seulement pour relever ce défi. Il s'est vu aux abords de la Seine, dans un café, avec un petit ensemble musical en arrière-fond. L'image est celle d'un homme seul qui s'ennuie devant son verre, triste et légèrement désabusé. Le texte est lent et doux; il amène une mélodie d'atmosphère soulignée du son du * flugelhorn *. 
Sur cette musique de «grill» ou de danse, l'interprète récite le texte plus qu'il ne le chante, on dirait une sorte de mélopée. Par ce récitatif (un peu comme l'aurait fait Francœur luimême), on se concentre davantage sur le texte. En écoutant cette version, Michel Rivard pense au Henri Salvador des années cinquante, tandis que Gerry Boulet songe à Boris Vian, à cause de son jazzé. Ces références nous transportent dans les caves de Saint-Germain-des-Prés et en plein existentialisme. Faulkner a donc choisi d'imager davantage le mot "Café", ce qui l'a éloigné de son style "country" habituel, auquel d'ailleurs s'attendaient les autres.

Gerry Boulet, lui, a insisté sur la situation d'attente, mais il ne joue pas pour autant sur la passivité. Il préfere, fidèle à sa nature, être plus affirmatif. La guitare aiguë, déchirante, telle une plainte, concorde avec sa voix blessée et cassée. L'émotion, plus retenue et intimiste chez Faulkner, devient, chez le rockeur, plus brute, plus proche de la sensation première et du cri. À cela, Lucien Francœur réagit: «La fille n’a plus le choix, elle est mieux de revenir!n

Ainsi, Boulet hausse le ton, module voix et musique, pour faire surgir et rugir un lyrisme dynamique. En ce sens, il se place davantage du côté de Rimbaud et du dérèglement des sens. La voix sort, sans fioritures, dans l'émotion pure. Du côté des variations apportées au texte par l'interprétation, Boulet insiste sur les mots "en t'attendant " en les répétant quelquefois pour en faire une obsession, une plainte lancinante. On est loin de la sensiblerie ou du fleur-bleue, sans s'écarter du romantisme. L'impatience, la fougue et la fébrilité dominent, soutenues par le mouvement et l'énergie rock, traduisant l'attente de l'amoureux aux abois.

Contrairement aux autres, François Cousineau imagine la musique entendue par celui qui attend au café. Ceci amène une distorsion entre la mélodie intérieure du personnage et la rythmique facile, un peu disco, dans laquelle baigne l'endroit. Ce procédé accentue la solitude du «je». Il semble impossible de bercer le mur indifférent, ce qui représente bien le spleen des fins de siècles. Dans sa composition, Cousineau a buté sur les mots "feutre ", qu'il trouvait difficile à traduire, et "Épinal ", dont il ne percevait pas le sens exact. Ce dernier passage, il l'a illustré d'une ligne plus classique, après avoir vérifié la signification, comme il a fait vivre la boîte à musique dans une autre strophe, pour prolonger le sens de ces expressions. 
80

Cette version, dans l'ensemble, focalise cependant moins sur le texte et sur le monde intérieur. C'est la voix chaude, modulée, "swignante" et jazzée de l'interprète qui ajoutera vie à la composition, puisque celle-ci est presque centralisée sur le décor de la scène. À cause du choix effectué par le compositeur, la chanson apparaît plus banale, moins * littéraire", car la musique représente celle que n'importe qui pourrait entendre, dans n'importe quel lieu d'attente.

Pour Michel Rivard, "crayon feutre" a été une pulsion d'écriture, a donné une couleur à sa mélodie. Avant de composer, il a vécu quinze jours avec le texte, sans écrire, l'apprenant par cœur, attendant le déclic. Le "feutre" l'a incité à aller dans un côté de lui qui lui était difficile à accepter (dit-il), celui de la douceur. Il a exploité alors une rime intérieure et fait des rejets, ce qui a accentué le rythme, tout comme les quelques répétitions qu'il a instaurées dans l'ensemble. Celles-ci donnent aussi l'illusion d'un texte plus long. On reconnaît bien là la façon de Michel Rivard qui donne la place au texte.

Il y a, dans cette version-ci, un petit soupçon "western " (comme souvent chez Beau Dommage et chez Rivard) qui donne de l'ampleur et souligne la solitude. En contrepartie, la section rythmique prend des petits accents tropicaux, ce qui ajoute de la légèreté, de la liberté et dédramatise la situation de séparation et d'attente vaine. Le spleen se fait donc plus verlainien. Les boucles effectuées par les rejets circonscrivent précisément l'univers clos du monde intérieur ici exposé. L'aspect français est mis en valeur par la manière qu'a le chanteur de prononcer le mot «radio», tandis que le côté poétique le sera par l'insistance sur le mot «Rimbaud ", souvent répété. Le modèle "chansonnier» peint donc cette interprétation, appuyé par la guitare acoustique et le ton intimiste. On songe aux tonalités de La complainte du phoque en Alaska.

Marie Bernard, elle, nous propose une vision plus ample et plus cosmique où les claviers électroniques, riches en textures, nous lancent dans le sidéral et dans l'universel. Il y a là à la fois quelque chose d'intellectuel et de très sensible, dans un heureux mélange: lire, par exemple, SPACE-OPERA (surexposition), poèmes de Roger DesRoches. Ces teintes musicales nous indiquent clairement que ce qui a présidé à cette version, c'est la posture même de la création proposée par le texte au moyen de ce personnage qui écrit dans son attente. 
À l'envolée musicale de cette version, la plus longue (quatre minutes quinze), le texte vient répondre, nous ramenant à la simplicité d'une "boîte à musique", des «images d'Épinal $*$ ou de l'émotion du «je *. Ce qui a frappé Marie Bernard, c'est aussi le présent de l'indicatif utilisé dans le texte, particulièrement le "j'écris *, souvent répété, qui donne l'action à la chanson et la priorité à l'acte créateur. On sent le temps passer et le geste qui vient s'y inscrire.

A contre-pied des autres compositeurs, tous masculins d'ailleurs, Marie Bernard utilise en quelque sorte les mots pour ponctuer sa musique. De plus, elle développe un aspect peu touché par les autres, celui du plaisir de créer qui serait l'aspect positif de ce moment de mélancolie. Pour montrer qu'il s'agit en réalité d'un espace de temps à habiter, elle détachera les strophes et puis chaque syllabe du mot "Épinal», se réfugiant davantage dans le « space * que dans l'« opéra *, ce qui explique le côté aérien et fait presque oublier le côté « café ".

Cette expérience, finalement, est passionnante, parce qu'elle dévoile différentes façons de lire un texte et aussi différentes façons de créer une chanson. Cet enregistrement et cette émission forment un document idéal, par exemple, pour les cours de création musicale ou littéraire ou pour enseigner la chanson. Et l'on voit, entre autres, comme l'a dit Francœur lui-même, que le texte idéal pour laisser inventer les autres, c'est un texte qui a quelque chose de minimal, de neutre, d'anodin, mais aussi une certaine concision, de même qu'une émotion concentrée et universelle. Ces caractéristiques sont celles mêmes qui ont permis au jury de faire un consensus, parce que, justement, chaque créateur y voyait quelques indices stimulants, mais aussi beaucoup de place pour sa propre liberté, son propre romantisme... et son propre crayon feutre! 